Philosophie ANTIQUE

\section{Philosophie antique}

Problèmes, Renaissances, Usages

19 | 2019

L'épicurisme antique

\title{
Simone vezzoli, Arcesilao di Pitane. L'origine del platonismo neoaccademico
}

Turnhout, Brepols, 2016 (Philosophie hellénistique et romaine), 300 p., ISBN 978-2-503-55029-9

Thomas Bénatouil

\section{CpenEdition}

\section{Journals}

Édition électronique

URL : https://journals.openedition.org/philosant/1936

DOI : 10.4000/philosant. 1936

ISSN : 2648-2789

Éditeur

Éditions Vrin

\section{Édition imprimée}

Date de publication : 31 octobre 2019

Pagination : 198-201

ISBN : 978-2-7574-2534-3

ISSN : 1634-4561

Référence électronique

Thomas Bénatouill, «Simone vezzol, Arcesilao di Pitane. L'origine del platonismo neoaccademico », Philosophie antique [En ligne], 19 | 2019, mis en ligne le 08 mai 2019, consulté le 02 décembre 2022. URL : http://journals.openedition.org/philosant/1936 ; DOI : https://doi.org/10.4000/philosant.1936

\section{(ब) $\Theta$}

Creative Commons - Attribution - Pas d'Utilisation Commerciale - Pas de Modification 4.0 International - CC BY-NC-ND 4.0

https://creativecommons.org/licenses/by-nc-nd/4.0/ 
savoir comment l'existence d'un dieu qui ne nous écoute pas peut avoir un effet positif sur l'être humain et sur sa conduite, mais aussi pourquoi il est si difficile, pour nous aujourd'hui, de répondre à cette question. Avec un geste dont la portée philosophique se révèle très intéressante, la réflexion sur la légitimité de l'emploi de catégories comme celles de « théologie », de « prière » et même de « dieu », dans le cadre des conceptions atomistes de la divinité, devient un miroir pour le lecteur qui se voit invité à réfléchir sur ces catégories mêmes et sur sa façon de les entendre. Le résultat de cette cinquième partie est la reconstitution de l'aspect le plus concret des théologies atomistes : une conception différente, nouvelle et «inattuale » de la relation à la divinité, dont la perfection et la béatitude apportent des bénéfices indirects à l'être humain : ce dernier s'en inspire et le

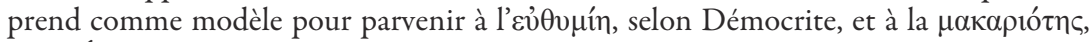
selon Épicure et ses disciples.

Cet ouvrage, riche et intéressant, parvient aux objectifs fixés dans son introduction. La cohérence et la solidité des différentes doctrines reconstituées démontrent toute la légitimité qu'il y a à considérer les réflexions atomistes sur le divin comme de véritables théologies, caractérisées par une remarquable portée éthique, et dont il est possible de reconstituer l'histoire. Cette histoire toutefois ne peut être reconstituée, nous rappelle l'auteur, qu'à condition de respecter les caractères particuliers des réflexions de chacun de ses protagonistes.

Simone Vezzoli, Arcesilao di Pitane. L'origine del platonismo neoaccademico, Turnhout, Brepols, 2016 (Philosophie hellénistique et romaine), 300 p., ISBN 978-2-503-55029-9.

Les travaux sur la Nouvelle Académie n'ont pas manqué durant les trente dernières années, mais très rares sont les monographies entièrement consacrées à l'un de ses trois grands représentants, l'exception qui confirme la règle étant l'ouvrage de Charles Brittain sur Philon de Larissa (Oxford, 2001). L'ouvrage de Simone Vezzoli (désormais S. V.) sur Arcésilas est issu d'une thèse de doctorat soutenue à Macerata en 2010 et a inauguré une nouvelle collection des éditions Brepols, Philosophie hellénistique et romaine (HФR), dirigée par C. Lévy et G. Reydams-Schils et qui compte trois ans plus tard déjà huit titres parus.

L'ouvrage de S. V. sur Arcésilas comprend une introduction, deux parties, Analisi (134 pages) et Fonti (122 pages), suivies par une bibliographie. Il ne comporte aucun index des passages, noms ou notions cités, ce qui est regrettable. La seconde partie recense l'ensemble des témoignages mentionnant Arcésilas; ils sont cités en grec ou latin, traduits en italien et assortis à chaque fois d'indications sur leur contexte, souvent d'un bref apparat critique et de renvois à d'autres témoignages parallèles ou à la littérature secondaire, voire d'une discussion détaillée dans deux ou trois cas controversés (par exemple le fameux passage de Plutarque, $A d v$. Col. 26-29, discuté p. 193-195). S. V. a réuni 161 témoignages et 10 dubia, mais plusieurs figurent sous deux numéros différents (mais ne sont cités qu'une seule fois par S. V.) s'ils nous ont été transmis par un auteur qui en cite un autre. Par exemple, les passages des Silles de Timon d'Athènes sur Arcésilas sont référencés d'abord au tout début des Fonti (F 2a à F 7), mais ne sont cités que dans les témoignages F 107, 115, 116 et 121, à savoir les passages de Diogène Läerce et Eusèbe (citant Numénius) qui citent les Silles. Les témoignages sont en effet présentés par S. V. dans l'ordre chronologique des sources. Cela permet de se faire une idée de la stratification 
historique des informations transmises sur Arcésilas, même si le fait que chaque passage ne soit cité qu'une fois empêche souvent de lire certains témoignages à leur place dans l'ordre chronologique, notamment les plus anciens (Timon, Ariston de Chios, Bion de Borysthène, etc.) qui ne nous sont connus que par des auteurs bien postérieurs (en particulier Diogène Laërce). On peut donc se demander si une organisation thématique des témoignages, accompagnée d'un index fontium ordonné chronologiquement, n'aurait pas été plus utile, et ce d'autant plus que les analyses thématiques de la première partie ne citent jamais les témoignages mais renvoient aux Fonti. Ces dernières ne prétendent en effet pas constituer « una vera et propria edizione (...) ma piuttosto un utile strumento per completare e approfondire le analisi precedentement svolte $\gg$ (p. 10). Précisons cependant que les Fonti de S. V. ont une valeur en elles-mêmes, indépendamment des Analisi, ne serait-ce que dans la mesure où elles citent dix-sept témoignages qui ne se trouvent pas dans le recueil des témoignages sur Crantor et Arcésilas de H. J. Mette (publié dans Lustrum, 26, 1984), par exemple De legibus I, 39 = F 37 (qui ferait selon S. V. allusion à une critique par Arcésilas et Carnéade de l'idée de loi naturelle, présentée juste avant par Cicéron, critique que S. V. analyse p. 77-78), ou trois passages de Sénèque sur des considérations ou conduites éthiques attribuées à Arcésilas (F 56-58).

Quant à la première partie, elle analyse d'abord les principaux aspects de la pensée d'Arcésilas (chapitre 1, p. 17-78), en commençant par sa méthode. Son usage de l'antilogie ad hominem s'appuierait sur le principe de l'isosthénie, même si ce terme pyrrhonien n'est pas attribué par nos sources à Arcésilas. Viennent ensuite les arguments épistémologiques puis éthiques d'Arcésilas. À leur sujet, une des principales questions posées par $S$. V. est celle, classique depuis Couissin, de savoir s'il défendait des positions in propria persona ou s'il ne faisait que les avancer dialectiquement à partir des thèses stoïciennes. S. V. déclare que les deux interprétations ne sont pas exclusives l'une de l'autre et souligne que la genèse dialectique de notions ou positions comme la suspension universelle, le caractère obscur (adelon) de toutes choses (p. 47-58) ou la réponse à l'objection de l'apraxia (p. 62-63) n'empêche pas qu'Arcésilas les ait assumées (sans pour autant en faire des dogmata). En ce qui concerne les arguments d'Arcésilas contre l'existence de la représentation compréhensive stoïcienne rapportés par Cicéron et Sextus, S. V. en propose une classification en trois catégories et examine chacune (p. 27-42). Il souligne à juste titre qu'Arcésilas s'en tient toujours au niveau de la représentation, sans rien avancer à propos de ses objets (p. 35) : il ne met donc pas en doute notre capacité d'atteindre la vérité mais celle de rendre la vérité évidente (p. 42), y compris quand il déclare les choses adela (p. 52-54). À propos des arguments éthiques d'Arcésilas en réponse à l'objection de l'apraxia, Plutarque et Sextus attribuent chacun à Arcésilas une réponse différente, l'une fondée sur le fait que l'assentiment n'est pas nécessaire à l'action, qui se porte « naturellement vers ce qui apparaît approprié (oikeion) », et l'autre fondée sur la notion d'eulogon, qui pourrait être d'origine stoïcienne. Pour éviter l'incohérence apparente entre ces deux réponses, $\mathrm{S}$. V. interprète de manière convaincante la première - rapportée par un passage très débattu de l'Adversus Colotem - comme portant sur le moment et non sur la possibilité même « d'une intervention discriminante de la raison dans la dynamique de l'action » (p. 72, je traduis). Arcésilas « se contente de décrire le mécanisme de l'action » et met en évidence que la raison ne peut intervenir que sur des « impulsions déjà développées » sur la base du désir du bonheur (p. 74). Cette intervention est quant à elle décrite par Sextus et peut donner lieu à une sagesse (phronesis) consciente de ses propres limites, qui nous guide vers le bonheur non de manière certaine, du fait de la suspension universelle, mais en suivant ce qui est eulogon (p. 68). 
Le second chapitre sur le «platonisme d'Arcésilas » (p. 79-148) a été présenté dès l'introduction comme inévitablement plus «spéculatif » (p. 10) du fait du manque de témoignages. Il constitue aussi la partie la plus originale de l'ouvrage de $S$. V. En effet, la thèse de l'inspiration platonicienne d'Arcésilas n'est pas nouvelle et a été en particulier réélaborée et développée par Carlos Lévy mais aussi sur certains points par Franco Trabattoni, deux savants dont $S$. V. suit explicitement les traces. Elle a cependant rarement été défendue de manière aussi complète et globale, c'est-à-dire en évitant des rapprochements seulement ponctuels ou « partiels » entre certains arguments néoacadémiciens et tel ou tel argument isolé à l'intérieur de l'œuvre de Platon (p. 92-93). En simplifiant, on pourrait dire que l'on peut défendre une telle interprétation globale en suivant deux voies (non exclusives l'une de l'autre), l'une qui met en évidence le platonisme implicite de la pensée d'Arcésilas, l'autre qui souligne la dimension aporétique de celle de Platon. S. V. commence par la première : il examine les différents arguments antiques en faveur de l'interprétation sceptique de Platon, montre leur justesse et les complète en soulignant (avec divers interprètes récents) que la forme dialoguée et le socratisme sont fondamentaux pour Platon. Arcésilas aurait juste privilégié la dimension non dogmatique sur la dimension psychagogique-éducative de cette pensée et pris au sérieux l'ironie socratique à l'égard du savoir (p. 100). À condition de noter que la forme dialoguée n'avait certainement pas pour Arcésilas les vertus intrinsèquement aporétiques que certains lecteurs contemporains lui prêtent, mais que c'est seulement son usage spécifique par Platon (par exemple pour présenter des thèses opposées sur un même sujet ou nuancer toute thèse) qui lui a permis de conjurer tout dogmatisme et aurait inspiré sa méthode à Arcésilas, cette interprétation est légitime et éclairante sur bien des points.

Moins convaincantes sont en revanche certaines tentatives de S. V. d'attribuer aux argumentations épistémologiques et éthiques antistoïciennes d'Arcésilas une inspiration directement platonicienne. Si les comparaisons de certaines objections à la représentation compréhensive avec le Sophiste (263d-264a) ou le Théétète sont tout à fait pertinentes, comment Arcésilas pourrait-il « adopter implicitement la perspective platonicienne selon laquelle la qualité de la connaissance dépend essentiellement de la nature de son objet » (p. 102, je traduis), alors qu'il a été bien montré dans le chapitre précédent qu'il ne raisonnait que du point de vue du contenu de nos représentations ? Si les objections à la représentation compréhensive sensible rapportées par Sextus, $A M$ VII, 411-415 font certes intervenir des défauts propres aux objets sensibles (p. 103), elles ne sont pas référées à Arcésilas. S. V. a défendu auparavant (p. 33) cette attribution en grande partie sur la base de l'hypothèse de l'inspiration platonicienne, ce qui rend son argumentation circulaire. Plus généralement, en voyant dans la suspension universelle ou l'obscurité promues par Arcésilas des ombres portées d'une conception positive mais idéale de la science (p. 116-118), voire d'une exigence de transcendance (p. 134), ne risque-t-on pas de sous-estimer l'originalité et la force d'une philosophie s'appuyant d'abord, voire seulement, sur l'exigence socratique négative (et acceptée par ses adversaires stoïciens) d'éviter la doxa ? Quant aux arguments éthiques, si on peut concéder à S. V. que le silence complet des témoignages sur leur possible inspiration platonicienne n'empêche pas de la rechercher (p. 135), on peut douter que la conception eudémoniste platonicienne du désir du bien par l'âme ait été mobilisée par Arcésilas, tant elle semble proche de celle des stoïciens. Même si l'on admet, ce qui me semble excessif, que ces derniers proposaient une « théorie instinctive de la pratique », où il existerait une « parfaite cohérence entre le souverain bien et la nature » (p. 140), comment Arcésilas pouvait-il le leur reprocher s'il défendait de son côté à la suite de Platon (et contre Zénon) l'idée que « l'impulsion à agir naît naturellement et nous conduit vers le bonheur » ? Quitte à chercher une 
inspiration platonicienne derrière les objections rapportées par le témoignage délicat de Plutarque, il vaudrait peut-être mieux aller chercher du côté de la division platonicienne de l'âme et de la position de la raison par rapport aux désirs qu'elle implique et qui est bien différente de celle de la raison stoïcienne.

Si les hypothèses les plus originales du livre de $S$. V. sur les sources platoniciennes d'Arcésilas susciteront donc certainement des discussions, cela ne diminue pas ses mérites : fournir un recueil accessible et une traduction italienne de tous les témoignages, proposer des bilans complets des débats interprétatifs sur les principaux témoignages et aspects de la pensée d'Arcésilas, qui présentent clairement les arguments en présence et offrent des solutions argumentées, généralement conciliatrices et souvent convaincantes, et mettre à l'épreuve l'idée d'une inspiration platonicienne du fondateur de la Nouvelle Académie.

Thomas BÉNATOUÏL

UMR Savoirs, Textes, Langage (CNRS/Univ. Lille) / Churchill College, University of Cambridge

Jula Wildberger, The Stoics and the State. Theory - Practice - Context, BadenBaden, Nomos, 2018 (Staatsverständnisse, 105), 263 p., ISBN 978-3-8487-2843-5.

La parution de l'ouvrage de Jula Wilberger doit être signalée comme un moment important des études stoïciennes. L'auteur nous livre là un travail de qualité, qui se propose de présenter de manière exhaustive la pensée politique stoïcienne, laquelle a fait l'objet de recherches ponctuelles, mais d'aucune monographie de cette ampleur. L'objectif est en effet d'enquêter à la fois sur le fonds conceptuel sous-jacent à cette théorie politique (la sociabilité humaine, les notions de droit, de loi, de justice et de nature), et sur ses développements pratiques au niveau cosmique comme au niveau constitutionnel, et ce, en couvrant assez largement l'histoire du stoïcisme, de ses origines zénoniennes jusqu'à ses développements les plus tardifs, ce qui inclut la reprise qu'en feront Juste Lipse, Kant et Nussbaum.

Le travail réalisé ici est remarquable, par sa précision érudite, la prise en compte de presque tous les résultats proposés jusqu'à présent (Schofield, Laurand, Jessop, Vogt), sa clarté et sa concision. Nul doute donc que cet ouvrage soit décidément utile. On regrettera toutefois que la période dite «Middle Stoicism » soit réduite à la portion congrue, ce qui est d'ailleurs souligné p. 169 (« a more detailed discussion of Middle Stoic political theory exceeds the scope of this book $\gg$ ), et que certains points importants aient fait l'objet d'un traitement trop rapide.

Étudier la pensée stoïcienne dans ses développements politiques pose une longue série de problèmes, qui naissent du fait premier que cette pensée apparaît fondamentalement réfractaire à la prise en charge de tout élément qui justement fait la politique. Étant donné que les paramètres politiques dans lesquels l'individu construit son action ne sont que des indifférents et non des biens préalables à l'obtention du bonheur, ni la revendication de droits, ni l'activisme consistant à améliorer, changer, ou même penser le régime politique en place, ne font partie, à première vue, de la panoplie stoïcienne. Et pourtant, ainsi que le rappelle JW au tout début de son ouvrage, on attribue aux stoïciens la paternité de deux idées fondamentales à toute théorie politique ultérieure : l'idée de loi naturelle et la proposition du cosmopolitisme. Comment comprendre alors précisément ce qui fait l'essence de la pensée politique stoïcienne ? L'ouvrage pose la question politique dans sa radicalité (p. 13) : peut-on encore penser un espace politique, si la cité est coextensive au cosmos, et si sa loi est celle de la nature ? 